\title{
X-ray data for the substitutional solid solution of the binary system ammonium dihydrogen phosphate with boric acid
}

\author{
M L N MADHU MOHAN and C HARANADH* \\ Department of Physics, Nagarjuna University, Nagarjunanagar 522 510, India \\ MS received 28 July 1993; revised 3 February 1994
}

\begin{abstract}
X-ray powder diffraction data for the binary system ammonium dihydrogen phosphate with boric acid is studied and it is inferred that the binary belongs to tetragonal system and forms a substitutional solid phase. On the basis of the space group the binary is predicted to exhibit electrooptic property along with piezoelectric and electrostriction properties. It is proposed that the phosphorus atom of ammonium dihydrogen phosphate is substituted by the boron atom of boric acid.
\end{abstract}

Keywords. X-ray diffraction; substitutional solid solution; tetragonal system.

\section{Introduction}

Ammonium dihydrogen phosphate $\left(\mathrm{NH}_{4} \mathrm{H}_{2} \mathrm{PO}_{4}, \mathrm{ADP}\right)$ belongs to the family $\mathrm{XH}_{2} \mathrm{PO}_{4}$. The other members of the family are $\mathrm{KH}_{2} \mathrm{PO}_{4}$ and $\mathrm{NaH}_{2} \mathrm{PO}_{4} ; \mathrm{NH}_{4} \mathrm{H}_{2} \mathrm{PO}_{4}$ and $\mathrm{KH}_{2} \mathrm{PO}_{4}$ belong to tetragonal system (Hellwege 1975). It has been reported that $\mathrm{KH}_{2} \mathrm{PO}_{4}$ and $\mathrm{NaH}_{2} \mathrm{PO}_{4}$ form a solid solution with amorphous boric acid $\left(\mathrm{H}_{3} \mathrm{BO}_{3}\right)$ (William 1958). We have studied the powder X-ray diffraction patterns of these tetragonal systems (Haranadh et al 1992). It was found that ADP formed a solid solution when mixed with boric acid. We have investigated the crystal system of this binary compound.

\section{Experimental}

Pure crystals of ADP and amorphous boric acid were weighed in equimolar proportions and the mixture was dissolved in triple distilled water. The weight fraction of ADP in the ADP/boric acid binary compound was 56.54\%. Crystals obtained were about $1 \mathrm{~mm}$ in dimensions and were colourless. For recording the $\mathrm{X}$-ray powder diffraction patterns a Seifert diffractometer was used with $\mathrm{Cu}-\mathrm{K}_{\alpha}$ radiation $(\lambda=$ $1.542 \AA)$. The density of the sample was determined by the flotation method. Birefringence was determined by the Babinet compensator technique using a $\mathrm{He}-\mathrm{Ne}$ laser.

\section{Results and discussion}

The X-ray data for the binary compound are presented in table 1 . Table 2 gives unit cell dimensions, unit cell volume, observed density, calculated density and the possible space groups of both pure ADP and the binary system of ADP/boric acid. From table 1 it is seen that for all reflections the observed values of $d$ (interplanar spacing) are in good agreement with those calculated. The maximum difference between $d_{\text {obs }}$ and $d_{\text {cal }}$ values was $0.73 \%$. It is inferred from the $\mathrm{X}$-ray data that the binary system 
Table 1. X-ray powder data.

\begin{tabular}{lccccccc}
\hline $\begin{array}{l}2 \theta \\
(\mathrm{deg})\end{array}$ & $h k l$ & $\begin{array}{c}d(\mathrm{obs}) \\
(\AA)\end{array}$ & $\begin{array}{c}d(\mathrm{cal}) \\
(\AA)\end{array}$ & $\begin{array}{c}2 \theta \\
(\mathrm{deg})\end{array}$ & $h k l$ & $\begin{array}{c}d(\mathrm{obs}) \\
(\AA)\end{array}$ & $\begin{array}{c}d(\mathrm{cal}) \\
(\AA)\end{array}$ \\
\hline 28.45 & 002 & 3.105 & 3.100 & 69.00 & 413 & 1.361 & 1.371 \\
33.30 & 121 & 2.674 & 2.672 & 70.30 & 422 & 1.335 & 1.336 \\
37.30 & 122 & 2.398 & 2.404 & 76.30 & 008 & 1.245 & 1.246 \\
38.51 & 104 & 2.317 & 2.317 & 78.45 & 510 & 1.215 & 1.215 \\
44.52 & 301 & 2.019 & 2.020 & 80.45 & 227 & 1.190 & 1.196 \\
57.15 & 226 & 1.609 & 1.609 & 87.45 & 416 & 1.112 & 1.116 \\
62.45 & 411 & 1.480 & 1.487 & 88.00 & 504 & 1.109 & 1.111 \\
67.30 & 315 & 1.387 & 1.387 & & & & \\
\hline
\end{tabular}

Table 2. Lattice parameters and crystal data.

\begin{tabular}{lcc}
\hline & ADP + Boric & ADP \\
\hline Crystál system & Tetragonal & Tetragonal \\
Cell dimensions $(\AA)$ & & \\
$\quad a$ & $6 \cdot 202$ & 7.500 \\
$b$ & $6 \cdot 202$ & 7.500 \\
$\quad c$ & $10 \cdot 000$ & 7.549 \\
Molecular weight & 88.415 & 115.03 \\
Number of molecules in unit cell $(z)$ & 4 & 4 \\
Observed density $\left(g / \mathrm{cm}^{3}\right)$ & 1.510 & 1.803 \\
Calculated density from X-ray data & $1 \cdot 526$ & 1.798 \\
$\quad\left(\mathrm{~g} / \mathrm{cm}^{3}\right)$ & 384.648 & 424.699 \\
Volume of unit cell $\left(\AA^{3}\right)$ & $\mathrm{P4} 2$ & $1 \overline{4} 2 \mathrm{~d}$ \\
Possible space group & & \\
\hline
\end{tabular}

of ADP/boric acid belongs to tetragonal system. The systematic absence of 001 for 1 odd uniquely determines the space group to be $\mathrm{P}_{2} 2$. The unit cell volume of the binary compound $\left(384.648 \AA^{3}\right)$ is less than that of the pure sample $\left(424.699 \AA^{3}\right)$.

ADP when mixed with boric acid forms a substitutional solid solution. Phosphorus in ADP is supposed to be replaced by boron. The atomic radii of phosphorus $(1 \cdot 11 \AA)$ and boron $(0.88 \AA)$ are nearly equal and this allows formation of a substitutional solid solution. Since an atom of higher atomic radius is being replaced by an atom of slightly lower atomic radius the volume of the unit cell of the binary compound is smaller compared to that of pure ADP. Similar behaviour has been reported earlier for the binary compound of $\mathrm{KH}_{2} \mathrm{PO}_{4}(\mathrm{KDP})$ with boric acid (Haranadh et al 1992).

The decrease in unit cell volume should be more for the ADP/boric acid binary compound compared to the KDP/boric acid binary $\left(287.5 \AA^{3}\right)$ compound, since the weight fraction of boric acid is higher by $10 \%$ in ADP/boric acid ( $56.54 \%$ boric acid) than in KDP/boric acid ( $36 \%$ boric acid). This large difference in the unit cell volumes can be attributed to the radii of ammonium radical and potassium atom. The radius of ammonium $(2 \cdot 184 \AA)$ is larger than that of the potassium atom $(2 \cdot 025 \AA)$. Thus the decrease in the unit cell volumes of binaries has been to some extent compensated by their respective radii of ammonium and potassium.

Similar behaviour is also expected in the case of $\mathrm{NaH}_{2} \mathrm{PO}_{4}$ /boric acid binary 
compound unit cell, since the atomic radius of sodium $(1.570 \AA)$ is much smaller compared to that of ammonium and potassium.

From knowledge of the tetragonal space groups we predict that the ADP/boric acid binary compound should exhibit electrooptic property (Kaminov 1974) along with electrostriction and piezoelectric properties (Narasimhamurty 1981). The number of independent electrostrictive coefficients is seven while the number of independent piezoelectric coefficients is one. The density of the binary ADP/boric acid compound, calculated by the flotation method as well as from the X-ray data are in good agreement.

Packing index is defined (Fairbairn 1943) as $\mathrm{PI}=\left(V_{\mathrm{a}} / V_{\mathrm{c}}\right) \times 10$, where $V_{\mathrm{a}}$ is the volume of the individual atoms in the unit cell and $V_{\mathrm{c}}$ is the volume of the unit cell of the crystal. Packing index of the ADP/boric acid binary compound is $2 \cdot 103$. This may be compared with that of pure ADP which is 1.235 . Thus it is concluded that the atoms in the binary are less compactly packed and loosely bound when compared to its pure sample. A similar decrease is observed in the case of $\mathrm{KDP} /$ boric acid binary compound, whose packing index is 6.416 whereas the packing index is 4.753 for the pure sample.

Birefringence of the ADP/boric acid binary compound has been measured in $a b$ plane using a Babinet compensator (Chavan and Patil 1985). The value of $\Delta n$ for $\mathrm{ADP} /$ boric acid at room temperature is measured to be 0.0152 while the value for pure ADP (Zernike Jr 1964) is 0.041. This decrease in birefringence supports the $\mathrm{X}$-ray data in which $a$ and $b$ values of the unit cell are smaller for ADP/boric acid compared to pure ADP, since birefringence is a bulk property.

\section{Conclusions}

(i) ADP/boric acid binary compound belongs to tetragonal system.

(ii) ADP/boric acid binary compound forms a substitutional solid solution.

(iii) In the binary compound of ADP/boric acid, phosphorus atom in ADP is substituted by boron atom of boric acid.

(iv) The volume of the unit cell is decreased because of the substitution of an atom with lower atomic radius (boron) by that of higher atomic radius (phosphorus).

\section{Acknowledgements}

The authors are grateful to the University of Hyderabad, Hyderabad for providing X-ray facility. One of the authors (MLNMM) acknowledges the financial support provided by CSIR, New Delhi.

\section{References}

Chavan S H and Patil R N 1985 Indian J. Pure Appl. Phys. 23101

Fairbairn H W 1943 Bull. Geol. Soc. Am. 541305

Haranadh C and Madhu Mohan M L N 1992 Bull. Mater. Sci. 14385

Hellwege K H 1975 Numerical data and functional relationships in technology (New York: Springer) p S135

Kaminov I V 1974 An introduction to electrooptic devices (New York: Academic Press) p 111

Narasimhamurty T S 1981 Photoelastic and electrooptic properties of crystals (New York: Plenum Press) pp 342,662

William F L 1958 Solubilities (New Jersey: Van Nostrand) p 276

Zernike Jr 1964 J. Opt. Soc. Am. 54121 